\title{
Breast Cancer Patient with Li-Fraumeni Syndrome: A Case Report Highlighting the Importance of Multidisciplinary Management
}

\author{
Beatriz Cirauqui $^{a}$ Teresa Morán ${ }^{a} \quad$ Anna Estival $^{a}$ Vanesa Quiroga ${ }^{a}$ \\ Olatz Etxaniz ${ }^{a} \quad$ Carmen Balana $^{a} \quad$ Matilde Navarro $^{b}$ Salvador Villàc \\ Rosa Ballester ${ }^{c}$ Mireia Margelía \\ a Medical Oncology Department, Badalona Applied Research Group in Oncology (B-ARGO \\ Group), Catalan Institute of Oncology, Badalona, Spain; ${ }^{b}$ Genetic Counseling Department, \\ Catalan Institute of Oncology, Badalona, Spain; 'Radiotherapy Oncology Department, \\ Catalan Institute of Oncology, Badalona, Spain
}

\section{Keywords}

TP53 - Li-Fraumeni syndrome - Breast cancer - Epidermal growth factor receptor-mutated adenocarcinoma $\cdot$ Glioblastoma

\begin{abstract}
Germline mutations in TP53, a tumor suppressor gene, are involved in the development of LiFraumeni syndrome, a rare disorder that predisposes carriers to multiple tumors. TP53 mutations have been associated with resistance to treatment and poor prognosis. A young female with the pathogenic germline TP53 mutation c.844C > T (p.R282W) was diagnosed with two metachronous breast tumors, one HER2-negative and the other HER2-positive. She was later diagnosed with synchronous glioblastoma, epidermal growth factor receptor-mutated lung adenocarcinoma, and HER2-negative breast cancer metastases. The patient was treated with local therapies, including brain surgery and radiotherapy, lung surgery, and a bilateral mastectomy, as well as with targeted systemic treatment. She proved to be highly sensitive to systemic therapy, and 13 years after the initial diagnosis of breast cancer and 6 years after the diagnosis of the two new primary tumors and recurrence of a prior cancer, she is alive with an excellent performance status. This surprising positive evolution may well be partly due to the pronged multidisciplinary approach to managing her disease and her extraordinary response to treatment: the lung adenocarcinoma showed excellent response to erlotinib; the breast cancer responded extremely well to eribulin and pegylated liposomal doxorubicin; and the glioblastoma has remained in response to surgery and radiotherapy. Despite harboring a TP53
\end{abstract}


mutation and having multiple tumors, this patient has shown an unexpectedly favorable evolution. The coordinated participation of a multidisciplinary team and the patient's own extraordinarily high sensitivity to systemic treatment played a major role in this evolution.

\section{Introduction}

Li-Fraumeni syndrome (LFS) is a rare, autosomal dominant, hereditary disorder that predisposes carriers to cancer development. It can affect several members of the same family, including young people and even infants, and leads to multiple primary tumors in the same individual [1]. Unlike other hereditary syndromes that predispose individuals to cancer, which are generally restricted to specific tumor sites, LFS can lead to the development of generally metachronous tumors at different sites. The tumors most frequently associated with LFS are soft tissue sarcomas, osteosarcoma, breast cancer, brain tumors, adrenocortical carcinoma, and leukemia. In 1990, two independent studies reported that germline mutations in TP53, a tumor suppressor gene, were the primary factor involved in the development of LFS [2,3]. TP53 mutations have been associated with resistance to treatment in some case reports and these patients are more likely to develop radiation-induced secondary malignancies $[4,5]$, which complicates disease management. The participation of a multidisciplinary team is crucial in determining the optimal course of treatment for these patients. Here we present the case of a young patient with LFS with several primary tumors and an unexpectedly good response to treatments and clinical outcomes.

\section{Case Report}

A 29-year-old female non-smoker had no history of major disease at the time of diagnosis. However, her father had died of lung cancer at the age of 60; her brother had died of astrocytoma at 31 years; her sister had undergone surgery for thymoma at 19 years of age and died at 30 of the spread of that tumor; her paternal aunt was diagnosed with breast cancer at the age of 30; and another paternal aunt had bilateral breast cancer and osteosarcoma. In March 2006, she was diagnosed with a cT2N0M0 right breast cancer carcinoma. In April 2006, the patient underwent a tumorectomy with a sentinel lymph node biopsy. She was diagnosed with grade 2 invasive ductal breast cancer $(1.5 \mathrm{~cm}$, estrogen receptor [ER]- and progesterone receptor [PR]-positive [95\%], human epidermal growth factor receptor 2 [HER2]-negative). There was no sentinel lymph node involvement (pT1N0) and no distant metastases. After cryopreservation of ovarian tissue, the patient entered the GEICAM 2003.02 clinical trial [6]. She was assigned to the experimental arm and received four cycles of 5-fluorouracil plus adriamycin plus cyclophosphamide (FAC) followed by 8 weeks of paclitaxel. She then received radiotherapy to the right breast and started hormonal therapy with tamoxifen, which was planned to continue for 5 years. Since the patient's age and family history suggested LFS, the patient was referred to the Genetic Counseling Unit of our hospital. An analysis of germline TP53 detected the pathogenic mutation c.844C > T. The analysis was performed in peripheral blood by PCR and Sanger sequencing, where the fraction of the pathogenic variant was approximately 50\%. In January 2009, a routine follow-up examination revealed a round, $12 \times 13 \times 22$ mm lump with irregular spiculated margins in some areas, located at the union of the lower quadrants of the left breast and an US-guided fine-needle aspiration biopsy revealed carcinoma. Positron emission tomography (PET) showed no further tumors. 
In February 2009, the patient underwent a bilateral subcutaneous mastectomy with an implant of the right nipple-areolar complex and preservation of the left nipple-areolar complex. A biopsy showing sentinel lymph node involvement was followed by a left axillary dissection isolating 11 lymph nodes. Histological analysis identified a second primary breast cancer consistent with a grade 2 invasive ductal breast cancer $(1.5 \mathrm{~cm}$, ER- and PR-positive [30\%], HER2-positive). Despite sentinel lymph node involvement, no other lymph node involvement was observed. The patient received adjuvant treatment with six cycles of docetaxel/carboplatin/trastuzumab (TCH), followed by maintenance trastuzumab, which continued as planned until April 2010. At the same time, she started hormonal therapy with anastrozole and luteinizing hormone-releasing hormone analogs and continued to be relapse-free.

In February 2012, the patient suffered a generalized tonic-clonic seizure. MRI of the brain revealed a 19-mm tumor in the left occipital lobe that could have been a metastasis or a highgrade glioma. A three-part diagnostic assessment was then performed. First, PET-computed tomography (CT) revealed a mass in the left upper lobe of the lung and bilateral non-characterizable subpleural nodules. Fine-needle aspiration biopsy of the lung tumor identified a primary lung adenocarcinoma with an epidermal growth factor receptor (EGFR) exon 19 deletion. Next, an MRI-assisted, neuronavigation-guided craniotomy with macroscopically complete excision of the left occipital lobe revealed grade 4 glioblastoma multiforme (GBM), without methylation of the 06-methylguanine DNA methyltransferase (MGMT) gene. Finally, a thoracoscopy of the right lung was performed, with resection of the tumor in the right lower lobe and excision of the diaphragmatic pleural nodules. Results showed metastases of the ductal carcinoma of the breast as microscopic (smallest $<2 \mathrm{~mm}$ ) subpleural nodules: cytokeratin 7 (CK7)-positive, ER+++, PR-focally positive, HER2-negative, thyroid transcription factor 1 (TTF1)-negative, and synaptophysin-negative. Faced with two new synchronous primary cancers as well as a recurrence of a prior breast cancer, our multidisciplinary team decided to focus treatment on the tumor with the worst prognosis - GBM - and initiated the standard treatment of radiotherapy plus temozolomide [7]. At the same time, since the patient's breast cancer was hormone-sensitive, without visceral involvement, and with a prolonged progression-free survival, we stopped anastrozole and started treatment with exemestane.

In June 2012, a follow-up thoracic examination revealed lung cancer progression. Since the origin of the progression was not clear, the patient stopped adjuvant temozolomide treatment and, in July 2012, underwent resection of the nodule in the left upper lobe, with biopsies of the pleural and lung tumors. The tumor in the left upper lobe was identified as invasive papillary lung adenocarcinoma $(4.4 \mathrm{~cm})$ with visceral pleural invasion and a $1-\mathrm{mm}$ resection margin. The analysis of the biopsies of the pleural and other lung tumors identified metastases of the primary breast cancer (CK7-positive, ER-positive, HER2-negative, TTF1negative) and the patient started treatment with capecitabine.

In January 2013, lytic bone metastases at T11 with epidural spinal cord compression and tumors at T2, T3 and T10 were detected, accompanied by pain. Radiotherapy was administered to $\mathrm{T} 11$, the patient started treatment with bisphosphonates, and the chemotherapy regimen was changed to oral vinorelbine. After completing three cycles of vinorelbine, in March 2013, a follow-up examination detected a left mediastinal mass, with lung and bone metastases. A CT-guided fine needle aspiration biopsy of the mediastinal mass showed metastases of the lung adenocarcinoma (ER-negative, TTF1-positive). Treatment was then modified to include radiotherapy (ten sessions at $30 \mathrm{~Gy}$ ) at T2 concurrent with paclitaxel and gemcitabine for breast cancer and erlotinib for lung cancer. The patient attained a complete response in the mediastinal metastases of the lung adenocarcinoma and a partial response in the lung and bone metastases of the breast cancer. Adverse events included grade 3 rash 

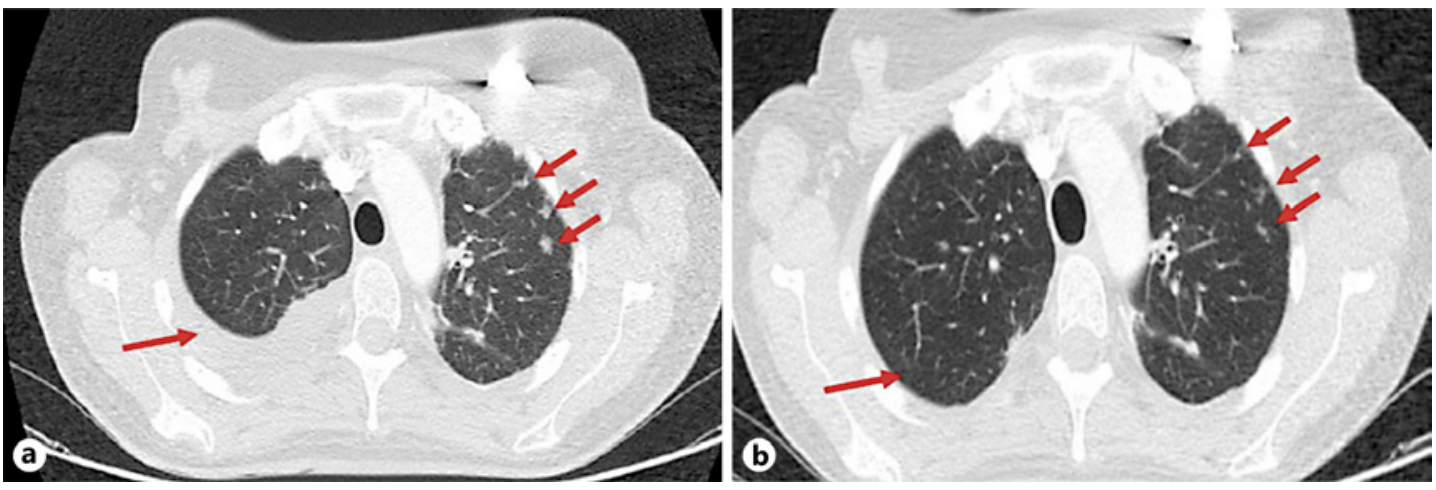

Fig. 1. CT of the thorax. Arrows indicate pleural effusion and lung metastases of breast cancer before starting eribulin treatment (a) and response after three cycles of eribulin (b).

related to erlotinib and grade 3 chemotherapy induced neurotoxicity that led to discontinuation of paclitaxel in January 2014. In April 2014, the patient complained of pleuritic chest pain and examination revealed the appearance of left-sided pleural effusion and bilateral lung opacities, the largest of which was $4 \mathrm{~mm}$ in the left upper lobe (Fig. 1a). The patient started treatment with eribulin $\left(1.23 \mathrm{mg} / \mathrm{m}^{2}\right.$, days 1 and 8, every 21 days) and also continued with erlotinib based on the lasting complete response of the mediastinal metastases. The patient attained a rapid partial response (Fig. 1b), which lasted until June 2016. Tolerability was excellent and she was able to continue treatment for a total of 35 cycles with no need for treatment interruption or dose adjustment.

In June 2016, new progression was observed in the lung and bone and the patient stopped treatment with eribulin and started pegylated liposomal doxorubicin. In August 2016, MRI of the brain detected progression of the GBM on surgical scar tissue, which was treated with hypofractionated focal radiotherapy with volumetric modulated arc therapy ( 5 Gy daily, 25 Gy total dose). The patient attained a partial response. At the time of writing, 13 years after the initial breast cancer diagnosis and 6 years after the diagnosis of synchronous breast cancer recurrence, advanced lung cancer, and GBM, the patient was continuing treatment with pegylated liposomal doxorubicin and erlotinib. The bone, pleura and lung metastases of the breast cancer were stable. The complete response in the primary lung adenocarcinoma was maintained. The GBM continued to have a partial response and was neurologically asymptomatic. The patient had an Eastern Cooperative Oncology Group (ECOG) performance status of 0 . Figure 2 shows the evolution of the patient over the course of these years.

\section{Discussion/Conclusion}

Patients with LFS generally have more than one primary tumor. In fact, $15-35 \%$ of cancer patients with LFS will develop multiple primary tumors over the course of their lives $[1,8]$. In the majority of cases, these tumors are metachronous and often related to radiotherapy administered to previous tumors [4,5]. Here we have presented the case of a young female patient with LFS who does not fit the typical description of these patients. Our patient had four primary tumors - two primary breast cancers, one GBM, and one lung adenocarcinoma - three of which (two primary cancers as well as recurrence of a prior breast cancer) were synchronous, making disease management more complex and requiring the coordination of a multidisciplinary team to decide on the best course of treatment. 


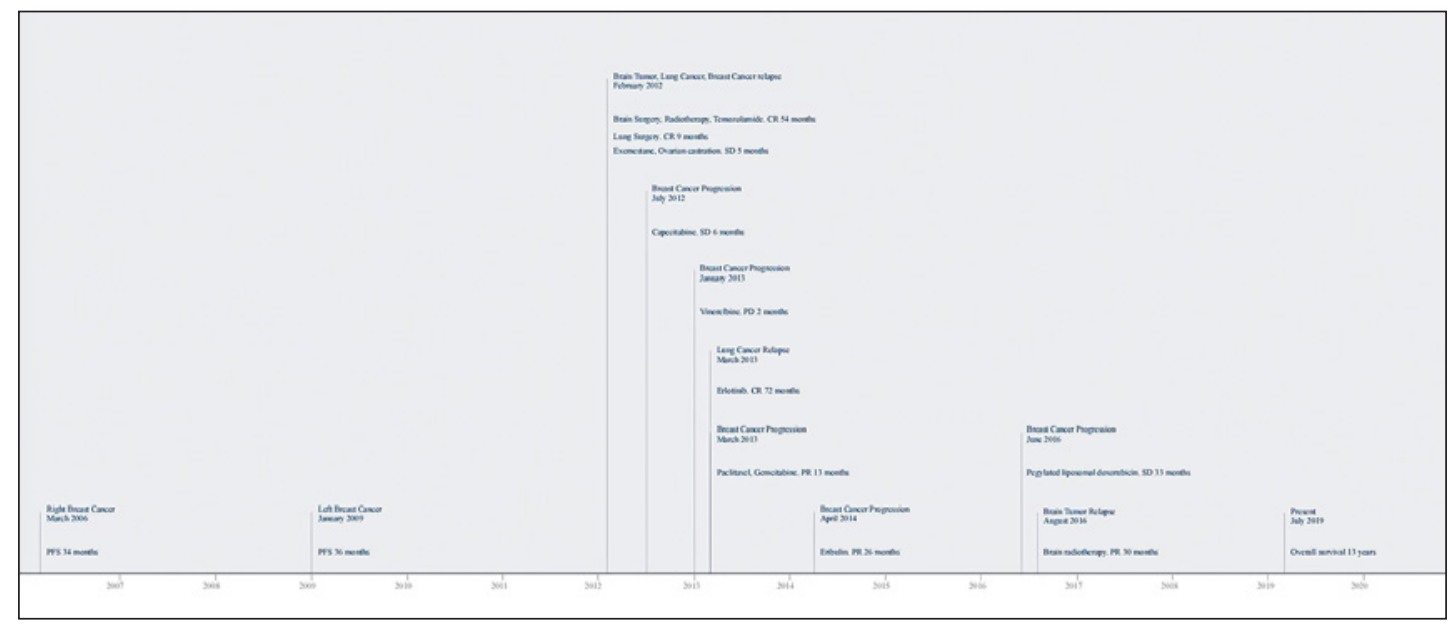

Fig. 2. Evolution of the patient. The patient obtained benefit from eribulin in the treatment of breast cancer, attaining progression-free survival of 26 months, and from erlotinib in the treatment of lung adenocarcinoma, attaining progression-free survival of 51 months. The patient's overall survival was 12 years from the diagnosis of the first breast cancer and 64 months from the diagnosis of synchronous glioblastoma, lung adenocarcinoma, and breast cancer recurrence. PFS, progression-free-survival; CR, complete response; PR, partial response; SD, stable disease; $\mathrm{PD}$, progression disease.

The analysis of germline TP53 detected the pathogenic mutation c.844C > T. It was performed in peripheral blood by PCR and Sanger sequencing, where the pathogenic variant fraction was approximately 50\%. Weber-Lassalle et al. [9] described three deleterious mutations in TP53 with low variant fractions detected in DNA from peripheral blood that could be attributable to clonal hematopoiesis induced by chemotherapy. The same authors considered that the germline variants showed a pathogenic variant fraction of $50 \%$ as it occurred in our patient. The diagnosis of LFS was not questioned at any time due to the positive result of the pathogenic TP53 mutation and the strong correlation with the neoplasms diagnosed in our patient. Also, TP53 mutation is really frequent in most tumors [10]. Consequently, studies of the mutational status in tumor tissue were not performed, but it would have been of interest to evaluate the TP53 mutation and p53 protein expression status in the different tumor samples.

Importantly, we were able to perform several biopsies on this patient, which helped to differentiate between new primary cancers and metastases of a prior tumor. It is of outmost importance to perform biopsies of as many tumors as possible in all cancer patients, but especially in patients with LFS, who are predisposed to multiple primary cancers. Biopsy results can help to detect potential therapeutic targets, which can improve treatment management and ultimately lead to a better prognosis for the patients. In our case, molecular analyses of the biopsy tissue identified HER2 amplification in the second primary breast cancer and a deletion in exon 19 of the EGFR exon 19 in the lung adenocarcinoma.

It has been postulated that prior exposure to chemotherapy can cause alterations in the EGFR or HER2 pathway [11-13]. Molecular alterations have been previously reported in patients with LFS [14], including rare HER2 mutations. The combination of TP53 mutations, a hallmark of LFS, together with EGFR mutations has been observed in 19\% of lung adenocarcinomas [15-18]. Our patient showed a high sensitivity to erlotinib, attaining a complete response in her lung adenocarcinoma. In general, the benefit of tyrosine kinase inhibitors, such as erlotinib, in patients with LFS is similar to the obtained benefit in other EGFR-mutated 
lung cancer patients, with a progression-free survival time of approximately 1 year. At the time of writing, however, our patient had remained progression-free for longer than expected (72 months).

As previously mentioned, the TPP53 mutation detected by PCR and Sanger sequencing was c.8444C $>\mathrm{T}$, a missense mutation that causes a change of the amino acid arginine to tryptophan at position 282 (p.R282W). This mutation has been reported in the IARC TP53 mutation database and in LOVD (Leiden Open Variation Database) as a pathogenic mutation. Given the death of the majority of the relatives, only a cosegregation study could be carried out in their two sisters. One was healthy and did not carry the mutation, and the second, affected by a malignant thymoma, carried the same mutation detected in our patient. A healthy paternal cousin was also studied and turned out not to be a carrier of the mutation. The IARC TP53 mutation database collects a large number of mutations in TP53, both somatic and germinal. Missense germ variants are the most frequent (73\%) and many of them have been described as pathogenic [19]. TP53 has a tumor suppressor function that can be activated by several cellular stresses, including DNA damage, and maintains genetic stability through different cellular functions including apoptosis and cell-cycle arrest. TP53 mutations cause not only the loss of their suppressor function acquiring a dominant negative activity, but also a gain in oncogenic properties that are independent of the function of the wild-type gene. Missense mutations promote tumorigenesis through mechanisms that interrupt response pathways to DNA damage [20]. The R282W mutant is associated with an earlier onset of familial cancers and poorer outcomes of cancer patients. Studies in non-small-cell cancer patients and chronic myelocytic leukemia patients also attested to its undesirable influence on the development and progression of cancer [21]. To some extent, the R282W loses some wild-type p53 tumor-suppressive activity. On the other hand, it may acquire oncogenic functions to regulate phenotypes such as cell growth, migration, invasion, metastasis, genomic instability and chemoresistance [22-24]. Our patient was diagnosed at an early age, as expected, but her survival has been better than expected, probably as a consequence of the excellent responses she has presented to different treatments. TP53 mutations are generally associated with a poor response to chemotherapy, hormonal therapy and radiotherapy in breast cancer patients. However, the predictive value of TP53 mutations is controversial [25]. Reactivation of tumors with altered TP53 can induce programmed cell death or senescence [26]. To the best of our knowledge, there have been no reports of an association between TP53 mutations and eribulin sensitivity. TP53 can influence sensitivity to antimicrotubules, such as eribulin, by regulating their composition and dynamics, suggesting that TP53 may not only be the guardian of the genome, but also the guardian of the cytoskeleton [27]. Our patient attained an extraordinarily rapid and long-lasting response of 26 months to eribulin, despite the fact that this was her fifth line of treatment for advanced disease and her fourth line of chemotherapy after prior progression to capecitabine, vinorelbine, and paclitaxel/ gemcitabine. The potential association between TP53 and paclitaxel sensitivity is not clear. Studies in cell lines have had diverse results, while the predictive value of TP53 in patients has not been demonstrated [28, 29]. Our patient responded well to paclitaxel, although treatment had to be stopped due to neurotoxicity. Since eribulin acts more selectively than vinorelbine, attaining a greater antitumor effect with smaller doses, we can speculate that the synergy between a chemotherapeutic agent and an antimicrotubule may depend on their specific mechanisms of action. Our patient also attained stable disease in her lung and bone metastases with pegylated liposomal doxorubicin. Moreover, her long progression-free survival of 26 months seems to belie the chemoresistance described in tumors with TP53 mutations [30]. Our patient also had an excellent evolution of her GBM, with a 6.5-year progression-free survival, which far exceeds the median overall survival of 13 months in patients with $>98 \%$ resection of the tumor [31]. According to the pivotal clinical trial results 
[7] that established the standard treatment, the median overall survival in patients with GBM receiving this treatment was 14.6 months. It is likely that the therapeutic arsenal used to treat the lung and breast cancers also impacted the evolution of the GBM. Several studies have examined the effect of EGFR tyrosine kinase inhibitors on GBM but with varying conclusions as to their efficacy [32-35]. Eribulin has shown antitumor activity in mouse models of GBM [36], but has not been tested in the clinical setting. We can speculate that chemosensitivity may increase in brain cancers as a result of damage to the blood-brain barrier by surgery and radiotherapy. We can also hypothesize that the excellent response of our patient may have been due to microvasculature remodeling and its anti-angiogenic effect, which is crucial in the development of GBM [37]. Finally, data in glioma cell lines indicate an association between the absence of functional TP53 and increased temozolomide sensitivity [38].

\section{Statement of Ethics}

The patient gave her signed informed consent to treatment. Approval by the Ethics Committee was waived as this was not a clinical study. The patient provided her signed informed consent to publication.

\section{Disclosure Statement}

B.C.: Consultant or advisory role: BMS, Roche, Merck; Speaking: BMS, Eisai, Roche; Travel grant: Roche, BMS, Eisai, Merck, Pierre-Fabre.

T.M.: Lectures and educational activities: Bristol-Myers Squibb, Roche, Lilly, Boehringer Ingelheim, Astra-Zeneca; Travel, Accommodations, Expenses: Roche, Astra Zeneca, Boehringer Ingelheim.

A.E.: Conferences expenses: Pharmamar/Roche/Lilly travel. Honoraria Roche.

V.Q.: Speaking: Roche, Eisai; Travel grant: BMS, Roche, Novartis, Pfizer Consultant or advisory role: Kern.

O.E., M.N., S.V., R.B.: no conflict of interest.

C.B.: grants from Instituto de Salud Carlos III; personal fees from Celgene, personal fees from Karyopharm, AbbVie, Pharmamar, Lipopharma.

M.M.: consultant or advisory role: Novartis, Pfizer, Roche, Kern: Speaking: Novartis. Founding Sources: Celgene, Kern.

\section{Funding Sources}

This study received no outside funding.

\section{Author Contributions}

B.C., T.M., A.E., V.Q., O.E., C.B., and M.M. coordinated the patient management and supervised the systemic therapy. M.N. supervised the genetic counseling of the patient. S.V. and R.B. supervised the radiotherapy. B.C. wrote the first draft of the manuscript. All authors participated in the critical review and revision of the manuscript and provided approval of the manuscript for submission. 
Cirauqui et al.: Breast Cancer Patient with Li-Fraumeni Syndrome

\section{References}

1 Hisada M, Garber JE, Fung CY, Fraumeni JF Jr, Li FP. Multiple primary cancers families with Li-Fraumeni syndrome. J Natl Cancer Inst. 1998 Apr 15;90(8):606-11.

2 Malkin D, Li FP, Strong LC, Fraumeni JF Jr, Nelson CE, Kim DH, et al. Germ line p53 mutations in a familial syndrome of breast cancer, sarcomas, and other neoplasms. Science. 1990 Nov 30;250(4985):1233-8.

3 Srivastava S, Zou ZQ, Pirollo K, Blattner W, Chang EH. Germ-line transmission of a mutated p53 gene in a cancer-prone family with Li-Fraumeni syndrome. Nature. 1990 Dec 20-27;348(6303):747-9.

4 Henry E, Villalobos V, Million L, Jensen KC, West R, Ganjoo K, et al. Chest wall leiomyosarcoma after breastconservative therapy for early-stage breast cancer in a young woman with Li-Fraumeni syndrome. J Natl Compr Canc Netw. 2012 Aug;10(8):939-42.

5 Yumrukcal F, Dirik Y, Cinar A, Eralp L. Fourth primary malignant tumor in a patient with possible li-fraumeni syndrome: synchronous diagnosis of postirradiation sarcoma, cutaneous relapse of a previous soft tissue sarcoma, and lung adenocarcinoma. Case Rep Orthop. 2014;2014:597207.

6 Martin M, Ruiz A, Ruiz Borrego M, Barnadas A, Gonzalez S, Calvo L, et al. Fluorouracil, doxorubicin, and cyclophosphamide (FAC) versus FAC followed by weekly paclitaxel as adjuvant therapy for high-risk, node-negative breast cancer: results from the GEICAM/2003-02 study. J Clin Oncol. 2013 Jul 10;31(20):2593-9.

7 Stupp R, Mason WP, van den Bent MJ, Weller M, Fisher B, Taphoorn MJ, et al. Radiotherapy plus concomitant and adjuvant temozolomide for glioblastoma. N Engl J Med. 2005 Mar 10;352(10):987-96.

8 Izawa N, Matsumoto S, Manabe J, Tanizawa T, Hoshi M, Shigemitsu T, et al. A Japanese patient with Li-Fraumeni syndrome who had nine primary malignancies associated with a germline mutation of the p53 tumorsuppressor gene. Int J Clin Oncol. 2008 Feb;13(1):78-82.

9 Weber- Lassalle K, Harter P, Hauke J, Ernst C, Kommoss S, Marmé F, et al. Diagnosis of Li-Fraumeni Syndrome: differentiating TP53 germline mutations from clonal hematopoiesis. Hum Mutat. 2018;39:2040-6.

10 TCGA Data Portal. Available from: https://tcga-data.nci.nih.gov.

11 Fortuno C, Cipponi A, Ballinger ML, Tavtigian SV, Olivier M, Ruparel V, et al. A quantitative model to predict pathogenicity of missense variants in the TP53 gene. Hum Mutat. 2019 Jun;40(6):788-800.

12 Yoshida A, Hayashi N, Suzuki K, Takimoto M, Nakamura S, Yamauchi H. Change in HER2 status after neoadjuvant chemotherapy and the prognostic impact in patients with primary breast cancer. J Surg Oncol. 2017 Dec;116(8):1021-8.

13 Wang Y, Ma X, Wei Y, Ma D, Gong P. Effect of platinum-based chemotherapy on EGFR gene mutation status in lung adenocarcinoma. Medicine. 2018 Jan;97(4):e9602.

14 Luo YH, Chen YM. Influence of chemotherapy on EGFR mutation status. Transl Lung Cancer Res. 2013 Dec; 2(6):442-4.

15 Serra V, Vivancos A, Puente XS, Felip E, Silberschmidt D, Caratu G, et al. Clinical response to a lapatinib-based therapy for a Li-Fraumeni syndrome patient with a novel HER2V659E mutation. Cancer Discov. 2013 Nov; 3(11):1238-44.

16 Bemis LT, Robinson WA, McFarlane R, Buyers E, Kelly K, Varella-Garcia M, et al. EGFR-mutant lung adenocarcinoma in a patient with Li-Fraumeni syndrome. Lancet Oncol. 2007 Jun;8(6):559-60.

17 Kosaka T, Yatabe Y, Endoh H, Kuwano H, Takahashi T, Mitsudomi T. Mutations of the epidermal growth factor receptor gene in lung cancer: biological and clinical implications. Cancer Res. 2004 Dec 15;64(24):8919-23.

18 Michalarea V, Calcasola M, Cane P, Tobal K, Izatt L, Spicer J. EGFR-mutated lung cancer in Li-Fraumeni syndrome. Lung Cancer. 2014 Sep;85(3):485-7.

19 Sugawara W, Arai Y, Kasai F, Fujiwara Y, Haruta M, Hosaka R, et al. Association of germline or somatic TP53 missense mutation with oncogene amplification in tumors developed in patients with Li-Fraumeni or Li-Fraumeni like syndrome. Genes Chromosomes Cancer. 2011;50:535-45.

20 Mir R, Zuberi M, Ahmad I, Javid J, Yadav P, et al. Biological and clinical implications of exon 8 P53 (R282W) gene mutation in relation to development and progression of chronic myeloid leukaemia patients in India population. J Cell Sci Ther. 2013;4:140.

21 Oren M, Rotter V. Mutant p53 gain-of-function in cancer. Cold Spring Harb Perspect Biol 2010;2:a001107.

22 Muller PA, Vousden KH, Norman JC. p53 and its mutants in tumor cell migration and invasion. J Cell Biol. 2011; 192:209-18.

23 Cooks T, Pateras IS, Tarcic O, Solomon H, Schetter AJ, Wilder S, et al. Mutant p53 prolongs NF- $\kappa$ B activation and promotes chronic inflammation and inflammation-associated colorectal cancer. Cancer Cell. 2013;23: 634-46.

24 Ricordel C, Labalette-Tiercin M, Lespagnol A, Kerjouan M, Dugast C, Mosser J, et al. EFGR-mutant lung adenocarcinoma and Li-Fraumeni syndrome: report of two cases and review of the literature. Lung Cancer. 2015 Jan;87(1):80-4.

25 Bertheau P, Plassa F, Espie M, Turpin E, de Roquancourt A, Marty M, et al. Effect of mutated TP53 on response of advanced breast cancers to high-dose chemotherapy. Lancet. 2002 Sep 14;360(9336):852-4.

26 Qian Y, Chen X. Senescence regulation by the p53 protein family. Methods Mol Biol. 2013;965:37-61.

27 Galmarini CM, Kamath K, Vanier-Viornery A, Hervieu V, Peiller E, Falette N, et al. Drug resistance associated with loss of p53 involves extensive alterations in microtubule composition and dynamics. Br J Cancer. 2003 Jun 2;88(11):1793-9. 
28 King TC, Akerley W, Fan AC, Moore T, Mangray S, Hsiu Chen M, et al. p53 mutations do not predict response to paclitaxel in metastatic nonsmall cell lung carcinoma. Cancer. 2000 Aug 15;89(4):769-73.

29 Malamou-Mitsi V, Gogas H, Dafni U, Bourli A, Fillipidis T, Sotiropoulou M, et al. Evaluation of the prognostic and predictive value of $\mathrm{p} 53$ and Bcl-2 in breast cancer patients participating in a randomized study with dosedense sequential adjuvant chemotherapy. Ann Oncol. 2006 Oct;17(10):1504-11.

30 Hientz K, Mohr A, Bhakta-Guha D, Efferth T. The role of p53 in cancer drug resistance and targeted chemotherapy. Oncotarget. 2017 Jan 31;8(5):8921-46.

31 Lacroix M, Abi-Said D, Fourney DR, Gokaslan ZL, Shi W, DeMonte F, et al. A multivariate analysis of 416 patients with glioblastoma multiforme: prognosis, extent of resection, and survival. J Neurosurg. 2001 Aug;95(2): 190-8.

32 Gallego O, Cuatrecasas M, Benavides M, Segura PP, Berrocal A, Erill N, et al. Efficacy of erlotinib in patients with relapsed gliobastoma multiforme who expressed EGFRVIII and PTEN determined by immunohistochemistry. J Neurooncol. 2014 Jan;116(2):413-9.

33 Haas-Kogan DA, Prados MD, Tihan T, Eberhard DA, Jelluma N, Arvold ND, et al. Epidermal growth factor receptor, protein kinase B/Akt, and glioma response to erlotinib. J Natl Cancer Inst. 2005 Jun 15;97(12): 880-7.

34 Mellinghoff IK, Wang MY, Vivanco I, Haas-Kogan DA, Zhu S, Dia EQ, et al. Molecular determinants of the response of glioblastomas to EGFR kinase inhibitors. N Engl J Med. 2005 Nov 10;353(19):2012-24.

35 Pelloski CE, Ballman KV, Furth AF, Zhang L, Lin E, Sulman EP, et al. Epidermal growth factor receptor variant III status defines clinically distinct subtypes of glioblastoma. J Clin Oncol. 2007 Jun 1;25(16):2288-94.

36 Towle MJ, Nomoto K, Asano M, Kishi Y, Yu MJ, Littlefield BA. Broad spectrum preclinical antitumor activity of eribulin (Halaven(R)): optimal effectiveness under intermittent dosing conditions. Anticancer Res. 2012 May; 3(5):1611-9.

37 Agoulnik SI, Kawano S, Taylor N, Oestreicher J, Matsui J, Chow J, et al. Eribulin mesylate exerts specific gene expression changes in pericytes and shortens pericyte-driven capillary network in vitro. Vasc Cell. 2014 Mar $1 ; 6(1): 3$.

38 Blough MD, Beauchamp DC, Westgate MR, Kelly JJ, Cairncross JG. Effect of aberrant p53 function on temozolomide sensitivity of glioma cell lines and brain tumor initiating cells from glioblastoma. J Neurooncol. 2011 Mar; 102(1):1-7. 